\title{
Patienten gut bewässert - Ärzte trocknen aus
}

Offensichtlich ist es für junge Ärzte auf der Intensivstation einfacher, die Flüssigkeitsbilanz ihrer Patienten aufrecht zu erhalten, als ihre eigene.

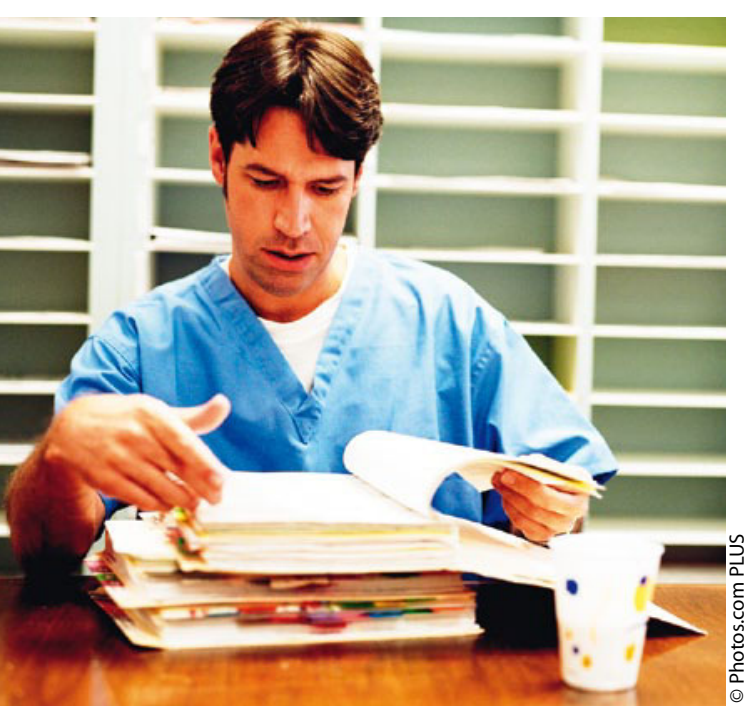

Kollege, trinken nicht vergessen!
- 19 junge Assistenzärzte ohne Nierenerkrankung erklärten sich bereit, zwischen dem 23. März und den 23. April 2009 das Urinvolumen zu dokumentieren, das sie während einer Schicht ausschieden.

Die Flüssigkeitsaufnahme lag im Ermessen des jeweiligen Arztes. Eine Oligurie war definiert als mittlere Urinausscheidung unter $0,5 \mathrm{ml} / \mathrm{kg}$ pro Stunde über $6 \mathrm{~h}$ hinweg oder länger. Bei 19 von 87 Schichten (22\%), in denen Urinmessungen vorlagen, erfüllten die Ärzte das Kriterium einer Oligurie und setzten sich dem Risiko eines akuten Nierenschadens aus. Bei 1\% der Schichten lagen bei den Ärzten die Kriterien eines akuten Nierenversagens vor.

Als Kontrollen dienten die Patienten der Intensivstation. Im Untersuchungszeitraum standen die täglichen Urinausscheidungen von 208 Patienten zur Verfügung. Die Wahrscheinlichkeit, dass die Ärzte eine Oligurie erlitten, lag fast doppelt so hoch wie bei den Patienten. Dennoch war die Mortalität unter den Ärzten erstaunlich niedrig und betrug $0 \%$. Junge Assistenzärzte auf der Intensivstation sind eben hart im Nehmen.

\section{Kommentar}

Die größte Schwäche der Studie besteht wohl darin, dass die Flüssigkeitsaufnahme der Ärzte nicht dokumentiert wurde, doch hätte das wohl die Möglichkeiten einer geschäftigen Intensivstation überschritten. Auch für die konsequente Sammlung des Urins durch die Ärzte wird man wohl keine Hand ins Feuer legen dürfen. Trotz der methodischen Mängel wirft die Untersuchung ein Schlaglicht auf die Arbeitsbelastung der jungen Ärzte auf Intensivstation. Die wichtigste Konsequenz ist wohl, dass das Personal sich gegenseitig ermahnt, das Trinken nicht zu vergessen.

H. S. FÜESSL "

\section{- A. W. Solomon et al.}

Urine output on an intensive care unit: casecontrol study. Brit. Med. J. 2010; 341: c6761

\section{Phantom-Vibrationen im Dienst}

Obwohl selten darüber gesprochen wird, sind sog. „Phantom-Vibrationen“ für die meisten diensthabenden Ärzte, die Pieper bei sich tragen, ein vertrautes Phänomen.

- Um nicht ständig durch Piepsgeräusche gestört zu werden, stellen viele Kollegen ihr Handy oder ihren Pieper auf den so genannten Vibrationsalarm. Viele Kollegen berichten allerdings über sensorische oder taktile Halluzinationen, d. h. sie spüren gelegentlich auch Vibrationen am Körper, obwohl das Gerät tatsächlich nicht angewählt wurde.

In einem akademischen Lehrkrankenhaus hat man nun 232 Ärzte und Pflegepersonal befragt, ob sie derartige Sensationen schon einmal an sich gespürt haben. $68 \%$ berichteten, dass sie dieses Phänomen kennen und bereits mindestens einmal an sich gespürt haben.

Vier Faktoren waren mit dem Auftreten von PhantomVibrationsempfindungen unabhängig assoziiert:

1. der Grad in der ärztlichen Hierarchie: Assistenzärzte erlebten das Phänomen um den Faktor 1,47 häufiger als Fachärzte oder Ärzte im Hintergrunddienst,
2. die Stelle, an der der Piper üblicherweise getragen wurde: beim Tragen in der Brusttasche empfanden die Personen derartige Empfindungen um den Faktor 1,66 häufiger als beim Tragen am Gürtel,

3. die Zeitdauer des Tragens: pro sechs Stunden Tragezeit kam es zu einer Zunahme der Häufigkeit um den Faktor 1,30 ,

4. überwiegender Einsatz des Gerätes im Vibrationsalarm.

\section{Kommentar}

Taktile Halluzinationen sind bei Trägern von Piepern und Handys anscheinend sehr häufig. Bei der hohen Prävalenz verwundert es, dass über dieses Phänomen so selten in der medizinischen Literatur berichtet wird. Untersuchenswert wäre auch, warum gerade junge Kollegen bei ihren ersten Diensten immer wieder die Funktion ihres Piepers überprüfen müssen - und warum dieses Phänomen bei Vorrücken in der ärztlichen Hierarchie deutlich nachlässt.

H. S. FÜESSL -

\section{- M. B. Rothberg et al.}

Phantom vibration syndrome among medical staff: a cross sectional survey. Brit. Med. J. 2010; 341: c6914 